\title{
A Systematic Review on School Air Quality and Its Impact on Student's Health in Tropical Countries
}

\author{
Bambang Wispriyono ${ }^{1,2}$, Budi Hartono ${ }^{1,2}$, Ririn Arminsih Wulandari ${ }^{1}$, Sasnila Pakpahan ${ }^{2}$, Gita Permata Aryati $^{2}$, \\ Nurmalasari $^{2} \&$ Okky Assetya $^{2}$ \\ ${ }^{1}$ Department of Environmental Health, Faculty of Public Health, Universitas Indonesia, Depok 16424, Jawa Barat, \\ Indonesia \\ ${ }^{2}$ Center of Industrial and Environmental Health Studies (PKKLI), Faculty of Public Health, Universitas Indonesia, \\ Depok 16424, Jawa Barat, Indonesia
}

Correspondence: Bambang Wispriyono, Department of Environmental Health, Faculty of Public Health, Universitas Indonesia, Depok 16424, Jawa Barat, Indonesia. Tel/Fax: 62-21-786-3479. E-mail: bwispri@ui.ac.id

\author{
Received: June 3, 2020 Accepted: July 21, 2020 Online Published: September 8, 2020 \\ doi:10.5539/gjhs.v12n11p20 URL: https://oi.org/10.5539/gjhs.v12n11p20
}

\begin{abstract}
Poor air quality in school areas has a negative impact on the student's health. Several studies in tropical countries have reported the risk exposure and environmental factors that were associated to the air quality in school areas. This paper presents a review of several case study research associated to air pollutants and environmental factors on the surrounding school environment and the health impact on students in tropical country. We selected and reviewed 18 research papers related to air quality in schools. The selection method was based on the inclusion and exclusion criteria. Throughout these studies, the most common source of air pollutants found in the classroom was particulate matter. Air quality in schools is affected by the distance between the school and the source of pollutants, ventilation, inhabitant, and season. Exposure to poor indoor air quality can increase health risk, respiratory problems, ocular problems, and students' absence from school.
\end{abstract}

Keywords: air pollutants, indoor air quality, school health, tropical

\section{Introduction}

Besides home, school is an important environment for children since they spend six to eight hours every day there. However, several researches focusing on the air quality at school suggested that poor air quality in the school environment could affect the students' health directly (e.g. respiratory problems) and indirectly (e.g. students absence from school) (Annesi-Maesano et al., 2013; Sasso et al., 2019). School children's age group is more vulnerable than adults to air pollution for several reasons, i.e., their higher respiration frequency, their higher physical activity, and their respiratory system is still developing (Annesi-Maesano et al., 2013).

The school air quality is related to the building condition, inhabitant, and surrounding environment. School buildings tends to have more inhabitants compared with office buildings, thus improper ventilation and building state could increase the risk of air pollution (Annesi-Maesano et al., 2013; Bo M et al., 2017; Zwoździak A et al., 2015). Because of its stable temperature, most of the school building in tropical country does not require heater and used natural ventilation instead. This allowed a higher air exchange rate and the outdoor air quality directly affecting the indoor air quality. Besides that, seasonal changes, temperature, and humidity variation may also affect the relationship between air pollution effects on health risk (Yap et al., 2019). In contrary with subtropical area, a tropical area has a wet and dry climate with a relatively stable temperature, which resulted in more stable annual air quality. This article aimed to study parameters and factors related to the school air quality and health impact on students in tropical countries.

\section{Methods}

We did a systematic article screening (Figure 1) on two online databases, i.e., Pubmed and Science Direct up to the second week of August 2019. We searched for research articles in English which were published during 2000 until 2019 by including keywords "air quality", "air pollution", "particulate matter", "nitrogen dioxide", "voc", "formaldehyde", "carbon dioxide", "carbon monoxide", "ozone", "hydrocarbon", and "school". We excluded the keywords "model" and "modeling" by using the advanced search option on those online databases. Next, we selected articles focused on tropical countries based on the information on the title and abstract. If this information were not available on the title and abstract, then the article was further excluded in the selection. We only studied 
research articles from the last ten years which directly measured air quality in school with school children as its subject, therefore we also screened the methods and subject of the research.

In this article, we extracted data from the results of research articles without further analyzing study site, subject, pollutant agent, influencing factors, and health impact. Several articles that we found did not include and/or analyze those variables. Related data from locations other than the school were also not included in this article.

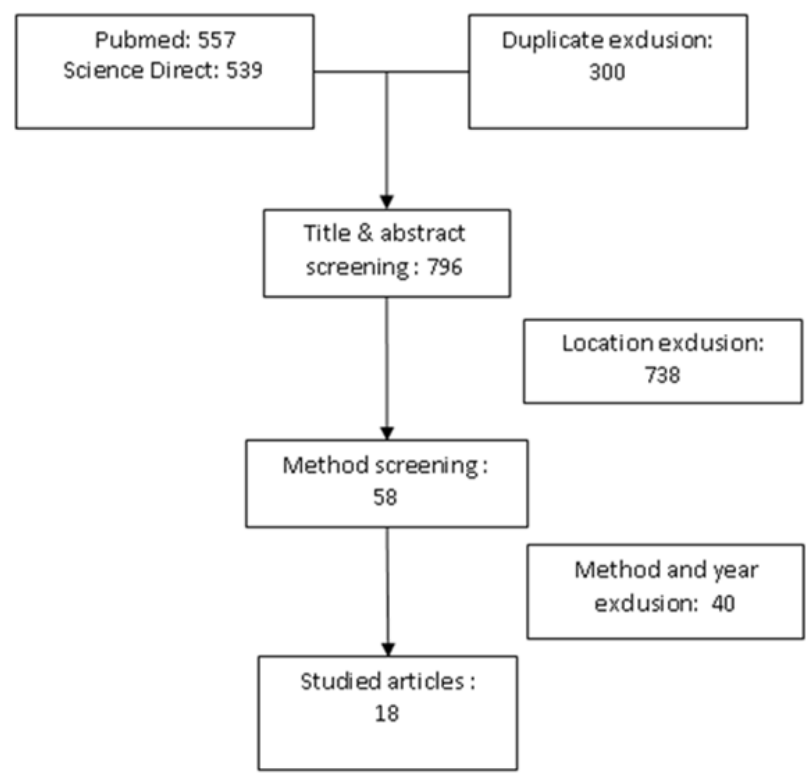

Figure 1. Research articles' screening scheme with the numbers of related articles

\section{Results}

\subsection{Articles Characteristics}

There were 18 articles selected in this study which were researches conducted in Malaysia (7), Brazil (6), and India (5). These studies focused on different school areas, i.e., preschool, primary, secondary, high school, and college. These schools were located in urban and suburban areas with different environmental characteristics that provide information on factors related to the school air quality. Several pollutants found which indicate the air quality were particulate matter, nitrogen oxide $\left(\mathrm{NO}_{2}\right)$, ozone $\left(\mathrm{O}_{3}\right)$, carbon dioxide $\left(\mathrm{CO}_{2}\right)$, carbon monoxide $(\mathrm{CO})$, sulfur dioxide $\left(\mathrm{SO}_{2}\right)$, volatile organic compounds (VOCs), polycyclic aromatic carbons (PAHs), allergens, and airborne microorganisms. Meanwhile, researches on the students' health impact from the pollutant exposure were discussed in 11 articles with the children from the investigated school being the most subject.

Most of the research that we studied used a cross-sectional method and quantitatively measured the exposure on the study site. In this article, we did not limit the research design used and the sample collection methods, however, we only used the quantitative and primary pollutant exposure data. Risk and health impact being the primary data were gathered through different methods.

\subsection{School Air Quality}

Based on 18 articles studied, particulate matter was being analyzed in 13 articles (67\%) from Malaysia, Brazil, and India. Other pollutants were $\mathrm{NO}_{2}, \mathrm{CO}_{2}, \mathrm{CO}, \mathrm{SO}_{2}, \mathrm{O}_{3}$, allergens, and microorganisms (fungi and bacteria). Besides that, chemical particulate compounds such as metal and ion were also analyzed in several articles to determine the source of pollutants. The methods used to measure air pollutants were varied and most of the measurement period used was 24 and 8 hours. We provide the data of air quality analysis from the 18 articles in Table 1.

\subsection{Health Impact}

Based on the data from Table 1, health analysis was studied by measuring health risk on the subject, respiratory function, ECP and FeNO, health history questionnaire, and students' complaints. Health problems reported were respiratory problems, eye problems, and symptoms of Sick Building Syndrome (SBS). The inflammation on the respiratory tract on students was indicated by the increase in Eosinophil Cationic Protein (ECP) and Fractional exhaled Nitric Oxide (FeNO). 
Table 1. Concentration of Air Pollutiont (2)

\begin{tabular}{|c|c|c|c|c|c|c|c|c|}
\hline \multirow{2}{*}{ Nr. } & \multirow{2}{*}{ Author (s) } & \multirow{2}{*}{ Study location (s)* } & \multirow{2}{*}{\multicolumn{2}{|c|}{ Subject }} & \multicolumn{2}{|l|}{ Air pollutant (s) } & \multirow{2}{*}{ Environmental factor (s) } & \multirow{2}{*}{ Health impact } \\
\hline & & & & & Indoor & Outdoor & & \\
\hline 1. & 6 & $\begin{array}{lr}\text { Primary } & \text { school (1), } \\
\text { Kuala } & \text { Lumpur, } \\
\text { Malaysia } & \end{array}$ & & & $\begin{array}{l}\mathrm{PM}_{2.5}(24 \text { hours }(\mathrm{h})) 11.2 \pm 0.45 \\
\mu \mathrm{g} / \mathrm{m}^{3} ; \mathrm{PM}_{2.5}(8 \mathrm{~h}) 10.2 \pm 0.45 \\
\mu \mathrm{g} / \mathrm{m}^{3} \\
\text { Others: metal }(\mathrm{Al}, \mathrm{Fe}, \mathrm{Zn}, \mathrm{Cr}, \mathrm{Cd} \text {, } \\
\mathrm{Ni}, \mathrm{Cu}) \text { and ion }\left(\mathrm{Ca}^{2+} \mathrm{Na}^{+}, \mathrm{K}^{+}, \mathrm{Ca}^{2+} \text {, }\right. \\
\left.\mathrm{Mg}^{2+}, \mathrm{Cl}^{-}\right)\end{array}$ & $\begin{array}{l}\mathrm{PM}_{2.5}(24 \mathrm{~h}) 11.4 \pm 0.44 \mu \mathrm{g} / \mathrm{m}^{3} \\
\mathrm{PM}_{2.5}(8 \mathrm{~h}) 8.35 \pm 0.85 \mu \mathrm{g} / \mathrm{m}^{3} \\
\text { Others: metal }(\mathrm{Al}, \mathrm{Fe}, \mathrm{Zn}, \mathrm{Cr}, \mathrm{Cd} \text {, } \\
\mathrm{Ni}, \mathrm{Cu}) \text { and ion }\left(\mathrm{Ca}^{2+}, \mathrm{Na}^{+}, \mathrm{K}^{+},\right. \\
\left.\mathrm{Ca}^{2+}, \mathrm{Mg}^{2+}, \mathrm{Cl}^{-}\right)\end{array}$ & $\begin{array}{l}\text { Location of school to the } \\
\text { source of pollutants } \\
\text { (traffic and industry), } \\
\text { students' activity, and the } \\
\text { building inhabitants }\end{array}$ & $\begin{array}{l}\text { Non carcinogenic health } \\
\text { risk } \\
\mathrm{HQ}<1 \text {, } \\
\text { Carcinogenic health risk } \\
\mathrm{CR}<1\end{array}$ \\
\hline 2. & 7 & $\begin{array}{ll}\text { School (1) and } \\
\text { university } \\
\text { Canoas, Brazil }\end{array}$ & & & $\begin{array}{l}\text { Chemical compound } \mathrm{PM}_{2.5}(3 \mathrm{~h}) \text { : } \\
\mathrm{Cr}, \mathrm{Fe}, \mathrm{Mn}, \mathrm{Ni}, \mathrm{Si}, \mathrm{Zn}, \mathrm{Pb}, \mathrm{Zr}, \mathrm{Ca} \text {, } \\
\mathrm{Cu}, \mathrm{K}, \mathrm{Cr} \text {, and } \mathrm{Cd}\end{array}$ & & $\begin{array}{l}\text { Location of school to the } \\
\text { source of pollutants } \\
\text { (traffic and industry), } \\
\text { indoor activities }\end{array}$ & \\
\hline 3. & 8 & $\begin{array}{l}\text { Secondary school (8), } \\
\text { Johor Baru, Malaysia }\end{array}$ & $\begin{array}{l}\text { Students } \\
(462, \\
14-16)\end{array}$ & age & $\begin{array}{l}\mathrm{CO}_{2}(8 \mathrm{~h}) 492 \mu \mathrm{g} / \mathrm{m}^{3} ; \mathrm{NO}_{2}(24 \mathrm{~h}) \\
24.3 \mu \mathrm{g} / \mathrm{m}^{3} ; \text { Formaldehyde }(24 \mathrm{~h}) \\
4.2 \mu \mathrm{g} / \mathrm{m}^{3} \text {; VOC }(4 \mathrm{~h}): \\
\text { - } \quad \text { Toluene } 17.5 \mu \mathrm{g} / \mathrm{m}^{3} \\
\text { - } \quad \text { Ethylbenzene } 1.9 \mu \mathrm{g} / \mathrm{m}^{3} \\
\text { - } \quad \text { Xylene } 85.4 \mu \mathrm{g} / \mathrm{m}^{3} \\
\text { - } \quad \text { Limonene } 5.4 \mu \mathrm{g} / \mathrm{m}^{3} \\
\text { - } \quad \text { Benzaldehyda } 2.0 \mu \mathrm{g} / \mathrm{m}^{3}\end{array}$ & $\begin{array}{l}\mathrm{CO}_{2}(8 \mathrm{~h}) 440 \mu \mathrm{g} / \mathrm{m}^{3} ; \mathrm{NO}_{2}(24 \mathrm{~h}) \\
23.8 \mu \mathrm{g} / \mathrm{m}^{3} ; \text { Formaldehyde }(24 \mathrm{~h}) \\
5.5 \mu \mathrm{g} / \mathrm{m}^{3} ; \text { VOC }(4 \mathrm{~h}): \\
\text { - } \quad \text { Toluene } 17.5 \mu \mathrm{g} / \mathrm{m}^{3} \\
\text { - } \quad \text { Ethylbenzene } 1.9 \mu \mathrm{g} / \mathrm{m}^{3} \\
\text { - } \quad \text { Xylene } 86.7 \mu \mathrm{g} / \mathrm{m}^{3} \\
\text { - } \quad \text { Limonene } 5.4 \mu \mathrm{g} / \mathrm{m}^{3} \\
\text { - } \quad \text { Benzaldehyde } 2.7 \mu \mathrm{g} / \mathrm{m}^{3}\end{array}$ & - & $\begin{array}{l}\text { Ocular and throat } \\
\text { symptoms, fatigue }\end{array}$ \\
\hline 4. & 9 & $\begin{array}{l}\text { Primary school (2), } \\
\text { Pune, India }\end{array}$ & Students & & $\begin{array}{l}\mathrm{PM}_{10}(8 \mathrm{~h}) 263.9 \mu \mathrm{g} / \mathrm{m}^{3} ; \mathrm{PM}_{2.5}(8 \mathrm{~h}) \\
135.8 \mu \mathrm{g} / \mathrm{m}^{3} ; \\
\mathrm{O}_{3}(8 \mathrm{~h}) 83.3 \mu \mathrm{g} / \mathrm{m}^{3} ; \mathrm{CO}(8 \mathrm{~h}) 268.4 \\
\mu \mathrm{g} / \mathrm{m}^{3} ; \mathrm{NO}_{2}(8 \mathrm{~h}) 46.7 \mu \mathrm{g} / \mathrm{m}^{3} ; \mathrm{SO}_{2} \\
(8 \mathrm{~h}) 9.2 \mu \mathrm{g} / \mathrm{m}^{3} ; \mathrm{CO}_{2}(8 \mathrm{~h}) 1249.11 \\
\mu \mathrm{g} / \mathrm{m}^{3} \\
\text { Others: metal }(\mathrm{Ca}, \mathrm{Mg}, \mathrm{Na}, \mathrm{K}, \mathrm{Fe} \text {, } \\
\mathrm{Al}, \mathrm{Ba}, \mathrm{Mn}, \mathrm{Cu}, \mathrm{Zn}, \mathrm{Co}, \mathrm{Pb}, \mathrm{Ni}, \mathrm{Cr} \text {, } \\
\text { and } \mathrm{Cd})\end{array}$ & $\begin{array}{l}\text { la } \\
\mathrm{PM}_{10}(8 \mathrm{~h}) 166.6 \mu \mathrm{g} / \mathrm{m}^{3} ; \mathrm{PM}_{2.5}(8 \mathrm{~h}) \\
88.9 \mu \mathrm{g} / \mathrm{m}^{3}\end{array}$ & 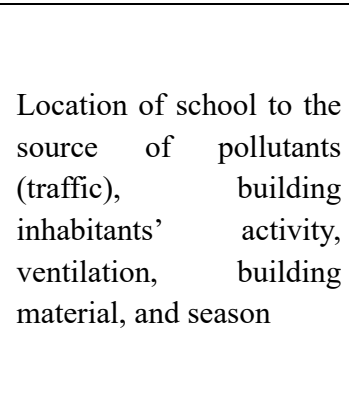 & $\begin{array}{l}\text { Cold, cough, fever, eye } \\
\text { and skin irritation, sore } \\
\text { throat, and sneezing }\end{array}$ \\
\hline
\end{tabular}




\begin{tabular}{|c|c|c|c|c|c|c|c|}
\hline 5. & 10 & $\begin{array}{l}\text { Secondary school (8), } \\
\text { Penang, Malaysia }\end{array}$ & $\begin{array}{l}\text { Students } \\
(368, \quad 14-16 \\
\text { year) }\end{array}$ & $\begin{array}{l}\text { DNA of Aspergillus versicolor } 15 \\
\text { copy } / \mathrm{g} \text { of dust, DNA of } \\
\text { Streptomyces } 7 \text { copy } / \mathrm{g} \text { of dust; } \mathrm{CO}_{2} \\
\left(50-70 \text { minutes): } 425 \mu \mathrm{g} / \mathrm{m}^{3}\right.\end{array}$ & $\mathrm{CO}_{2}(1 \mathrm{~h}): 407 \mu \mathrm{g} / \mathrm{m}^{3}$ & Humidity & $\begin{array}{l}\text { Wheeze, current asthma, } \\
\text { airway infection, and } \\
\text { increase level of FeNO (> } \\
20 \mathrm{ppb})\end{array}$ \\
\hline 6. & 11 & $\begin{array}{l}\text { Primary school (3) } \\
\text { and residences, Quito, } \\
\text { Brasil }\end{array}$ & & $\begin{array}{l}\underline{\text { School }} \\
\mathrm{PM}_{10}(7 \text { days }) 19.6 \pm 13.6-26.7 \pm \\
15.9 \mu \mathrm{g} / \mathrm{m}^{3} ; \mathrm{PM}_{10-2,5}(7 \text { days }) 5.73 \pm \\
2.8-16.1 \pm 11.6 \mu \mathrm{g} / \mathrm{m}^{3} ; \mathrm{PM}_{2,5}(7 \\
\text { days }) 10.6 \pm 4.9-14.7 \pm 15.6 \mu \mathrm{g} / \mathrm{m}^{3} \\
\underline{\text { Residence }} \\
\mathrm{PM}_{10}(7 \text { days }) 33.1 \pm 14.5-45.3 \pm \\
34.8 \mu \mathrm{g} / \mathrm{m}^{3} ; \mathrm{PM}_{10-2,5}(7 \text { days }) 12.4 \pm \\
7.9-16.9 \pm 21.1 \mu \mathrm{g} / \mathrm{m}^{3} ; \mathrm{PM}_{2,5}(7 \\
\text { days }) 19.3 \pm 14.6-29.0 \pm 30.5 \\
\mu \mathrm{g} / \mathrm{m}^{3}\end{array}$ & $\begin{array}{l}\underline{\text { School }} \\
\mathrm{PM}_{10}(7 \text { days }) 20.7 \pm 3.7-24.2 \pm \\
13.1 \mu \mathrm{g} / \mathrm{m}^{3} ; \mathrm{PM}_{10-2,5}(7 \text { days }) 12.4 \pm \\
7.9-16.9 \pm 21.1 \mu \mathrm{g} / \mathrm{m}^{3} ; \mathrm{PM}_{2,5}(7 \\
\text { days }) 10.9 \pm 3.2-13.2 \pm 3.5 \mu \mathrm{g} / \mathrm{m}^{3} \\
\underline{\text { Residence }} \\
\mathrm{PM}_{10}(7 \text { days }) 21.1 \pm 7.3-25.1 \pm \\
13.2 \mu \mathrm{g} / \mathrm{m}^{3} ; \mathrm{PM}_{10-2,5}(7 \text { days }) 8.2 \pm \\
2.4-11.6 \pm 6.6 \mu \mathrm{g} / \mathrm{m}^{3} ; \mathrm{PM}_{2,5}(7 \\
\text { days }) 14.3 \pm 10.1-12.5 \pm 14.6 \\
\mu \mathrm{g} / \mathrm{m}^{3}\end{array}$ & $\begin{array}{l}\text { Location of school to the } \\
\text { source of pollutants } \\
\text { (traffic), ventilation }\end{array}$ & \\
\hline 7. & 12 & $\begin{array}{l}\text { Primary school (2), } \\
\text { Kuala Lumpur and } \\
\text { Putrajaya, Malaysia }\end{array}$ & & 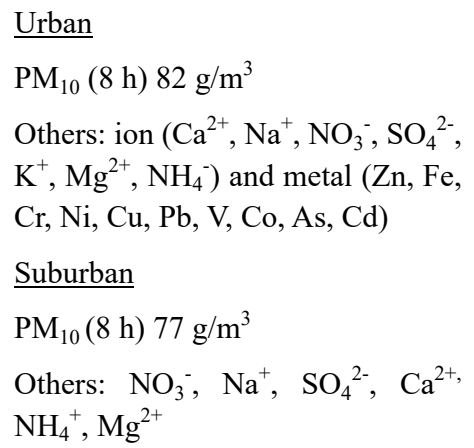 & $\begin{array}{l}\text { Urban } \\
\mathrm{PM}_{10}(8 \mathrm{~h}) 77 \mathrm{~g} / \mathrm{m}^{3} \\
\text { Others: ion }\left(\mathrm{Na}^{+}, \mathrm{NH}_{4}^{+}, \mathrm{K}^{+}, \mathrm{NO}_{3}^{-}\right) \\
\text {and metal }(\mathrm{Zn}, \mathrm{Cr}, \mathrm{Co}, \mathrm{Cu}, \mathrm{Ni}) \\
\text { Suburban } \\
\text { Others: ion }\left(\mathrm{SO}_{4}^{2-}, \mathrm{Na}^{+}, \mathrm{NH}_{4}^{+} \text {, }\right. \\
\left.\mathrm{Mg}^{2+}\right) \text { and metal }(\mathrm{Cd}, \mathrm{Cr})\end{array}$ & $\begin{array}{l}\text { Location of school to the } \\
\text { source of pollutants } \\
\text { (traffic, construction site, } \\
\text { biomass incineration, } \\
\text { industry, and sea), } \\
\text { building inhabitants' } \\
\text { activity, and building } \\
\text { material }\end{array}$ & $\begin{array}{l}\text { Non-carcinogenic health } \\
\text { risk } \\
\mathrm{HQ}>1\end{array}$ \\
\hline 8. & 13 & $\begin{array}{l}\text { Preschool (2), } \\
\text { Selangor, Malaysia }\end{array}$ & $\begin{array}{l}\text { Students }(98, \\
5-6 \text { years) }\end{array}$ & $\begin{array}{l}\underline{\text { Industry }} \\
\mathrm{PM}_{10}(4 \mathrm{~h}) 126.00 \mu \mathrm{g} / \mathrm{m}^{3}, \mathrm{VOCs}(4 \\
\text { h) } 0.02 \mathrm{ppm} \text {, fungi } 361.00 \mathrm{CFU} / \mathrm{m}^{3} \text {; } \\
\text { and bacteria } 275.00 \mathrm{CFU} / \mathrm{m}^{3} \\
\underline{\text { Suburban }} \\
\mathrm{PM}_{10}(4 \mathrm{~h}) 126.00 \mu \mathrm{g} / \mathrm{m}^{3}, \mathrm{VOCs}(4 \\
\text { h) } 0.02 \mathrm{ppm} \text {, fungi } 361.00 \mathrm{CFU} / \mathrm{m}^{3} \text {; } \\
\text { and bacteria } 275.00 \mathrm{CFU} / \mathrm{m}^{3}\end{array}$ & - & $\begin{array}{l}\text { Location of school to the } \\
\text { source of pollutants } \\
\text { (traffic) }\end{array}$ & $\begin{array}{l}\text { Increase the risk of } \\
\text { respiratory problems } \\
\text { Industry: ECP } 1.37 \\
\text { ng/mL } \\
\text { Suburban: } 0.66 \mathrm{ng} / \mathrm{mL}\end{array}$ \\
\hline
\end{tabular}




\begin{tabular}{|c|c|c|c|c|c|c|c|}
\hline \multirow[b]{2}{*}{9.} & \multirow[b]{2}{*}{14} & \multirow[b]{2}{*}{$\begin{array}{l}\text { Schools } \\
\text { Agra,India }\end{array}$} & \multirow[b]{2}{*}{$\begin{array}{l}\text { Students } \\
(300)\end{array}$} & $\underline{\text { Roadside }}$ & $\underline{\text { Roadside }}$ & \multirow[b]{2}{*}{$\begin{array}{l}\text { Location of school to the } \\
\text { source of pollutants } \\
\text { (traffic, building } \\
\text { inhabitants, activity, } \\
\text { ventilation) }\end{array}$} & \multirow[b]{2}{*}{$\begin{array}{l}\text { Asthma or respiratory } \\
\text { problems, cold, flu, } \\
\text { itching, eye irritation, } \\
\text { shortness of breath, } \\
\text { headache, and dizziness }\end{array}$} \\
\hline & & & & $\begin{array}{l}\mathrm{PM}_{10}(6 \mathrm{~h}) 264.91 \pm 33.73 \mu \mathrm{g} / \mathrm{m}^{3} \\
\mathrm{PM}_{2.5}(6 \mathrm{~h}) 81.85 \pm 13.06 \mu \mathrm{gg} / \mathrm{m}^{3} \\
\mathrm{PM}_{1,0}(6 \mathrm{~h}) 55.43 \pm 11.12 \mu \mathrm{g} / \mathrm{m}^{3} \\
\text { Others: } \mathrm{Mg}^{2+}>\mathrm{Ca}^{2+}>\mathrm{K}^{+}>\mathrm{Na}^{+}> \\
\mathrm{NO}_{3}^{-}>\mathrm{SO}_{4}{ }^{2-}>\mathrm{Cl}^{-}>\mathrm{F}^{-} \\
\text {Residence } \\
\mathrm{PM}_{10}(6 \mathrm{~h}) 261.11 \pm 45.48 \mu \mathrm{g} / \mathrm{m}^{3} ; \\
\mathrm{PM}_{2.5}(6 \mathrm{~h}) 78.33 \pm 12.44 \mu \mathrm{g} / \mathrm{m}^{3} ; \\
\mathrm{PM}_{1,0}(6 \mathrm{~h}) 53.20 \pm 9.59 \mu \mathrm{g} / \mathrm{m}^{3} \\
\mathrm{Others}^{3} \mathrm{Mg}^{2+}>\mathrm{Ca}^{2+}>\mathrm{K}^{+}>\mathrm{NO}_{3}{ }^{-}> \\
\mathrm{Na}^{+}>\mathrm{SO}_{4}{ }^{2-}>\mathrm{Cl}^{-}>\mathrm{F}^{-}\end{array}$ & $\begin{array}{l}\mathrm{PM}_{10}(6 \mathrm{~h}) 240.96 \pm 38.08 \mu \mathrm{g} / \mathrm{m}^{3} ; \\
\mathrm{PM}_{2.5}(6 \mathrm{~h}) 75.14 \pm 16.37 \mu \mathrm{g} / \mathrm{m}^{3} \\
\mathrm{PM}_{1,0}(6 \mathrm{~h}) 51.48 \pm 12.19 \mu \mathrm{g} / \mathrm{m}^{3} \\
\text { Others: } \mathrm{Mg}^{2+}>\mathrm{Ca}^{2+}>\mathrm{K}^{+}>\mathrm{NO}_{3}^{-}> \\
\mathrm{Na}^{+}>\mathrm{SO}_{4}{ }^{2-}>\mathrm{Cl}^{-}>\mathrm{F}^{-} \\
\text {Residence } \\
\mathrm{PM}_{10}(6 \mathrm{~h}) 235.51 \pm 34.48 \mu \mathrm{g} / \mathrm{m}^{3} \\
\mathrm{PM}_{2.5}(6 \mathrm{~h}) 71.95 \pm 14.55 \mu \mathrm{g} / \mathrm{m}^{3} ; \\
\mathrm{PM}_{1,0}(6 \mathrm{~h}) 48.62 \pm 11.97 \mu \mathrm{g} / \mathrm{m}^{3} \\
\mathrm{Others}^{3} \mathrm{Mg}^{2+}>\mathrm{Ca}^{2+}>\mathrm{K}^{+}>\mathrm{NO}_{3}^{-}> \\
\mathrm{SO}_{4}{ }^{2-}>\mathrm{Na}^{+}>\mathrm{Cl}^{-}>\mathrm{F}^{-}\end{array}$ & & \\
\hline 10. & 15 & $\begin{array}{l}\text { Preschool (30), } \\
\text { Araraquara, Brazil }\end{array}$ & $\begin{array}{l}\text { Students } \\
(750, \quad 4-5 \\
\text { years })\end{array}$ & TSP & - & $\begin{array}{lr}\text { Pre-harvest } & \text { burning } \\
\text { activity } & \text { (biomass } \\
\text { burning) } & \end{array}$ & $\begin{array}{l}\text { Respiratory problems } \\
\text { which impacted on } \\
\text { students absence in } \\
\text { school }\end{array}$ \\
\hline 11. & 16 & $\begin{array}{l}\text { Secondary school (3), } \\
\text { Bandar Baru Bangi } \\
\text { and } \\
\text { Malaysia }\end{array}$ & & $\begin{array}{l}\mathrm{PM}_{10}(8 \mathrm{~h}) 31 \mu \mathrm{g} / \mathrm{m}^{3} ; \\
\mathrm{PM}_{2.5}(8 \mathrm{~h}) 18 \mu \mathrm{g} / \mathrm{m}^{3} ; \\
\mathrm{PM}_{1}(8 \mathrm{~h}) 16 \mu \mathrm{g} / \mathrm{m}^{3} ; \\
\mathrm{CO}_{2}(8 \mathrm{~h}) 502 \mathrm{ppm} ; \\
\mathrm{CO}(8 \mathrm{~h}) 0.3 \mathrm{ppm}\end{array}$ & $\begin{array}{l}\mathrm{PM}_{10}(8 \mathrm{~h}) 46 \mu \mathrm{g} / \mathrm{m}^{3} ; \\
\mathrm{PM}_{2.5}(8 \mathrm{~h}) 38 \mu \mathrm{g} / \mathrm{m}^{3} ; \\
\mathrm{PM}_{1}(8 \mathrm{~h}) 35 \mu \mathrm{g} / \mathrm{m}^{3} ; \\
\mathrm{CO}_{2}(8 \mathrm{~h}) 486 \mathrm{ppm} ; \\
\mathrm{CO}(8 \mathrm{~h}) 0.8 \mathrm{ppm}\end{array}$ & $\begin{array}{l}\text { Location of school to the } \\
\text { source of pollutants } \\
\text { (traffic, industry), } \\
\text { building inhabitants' } \\
\text { activity, temperature, } \\
\text { humidity }\end{array}$ & \\
\hline 12. & 17 & $\begin{array}{l}\text { Primary school (1), } \\
\text { Delhi, India }\end{array}$ & & - & $\begin{array}{l}\mathrm{PM}_{10}(24 \mathrm{~h}) 222.2 \pm 111.3 \mu \mathrm{g} / \mathrm{m}^{3} \\
\mathrm{PAH} 103.3 \pm 50.4 \mathrm{ng} / \mathrm{m}^{3}\end{array}$ & Location and season & $\begin{array}{l}\text { Carcinogenic health risk } \\
\text { PAH ILCR } \\
3.18 \times 10^{-6}\end{array}$ \\
\hline 13. & 18 & $\begin{array}{l}\text { Secondary school (8), } \\
\text { Johor } \quad \text { Bahru, } \\
\text { Malaysia }\end{array}$ & $\begin{array}{l}\text { Students } \\
(462, \quad 14-16 \\
\text { years })\end{array}$ & $\begin{array}{l}\mathrm{CO}_{2}(50-70 \text { minutes) } 380-690 \mathrm{ppm} ; \\
\mathrm{LPS} 40.62 \mathrm{nmol} / \mathrm{g} ; \mathrm{MUA} 23.39 \\
\mu \mathrm{g} / \mathrm{g} ; \quad \text { Ergosterol 2.66 } \mu \mathrm{g} / \mathrm{g} \text {; } \\
\text { allergens from cats (Fel d1), dogs } \\
\text { (Can f 1), or horses (Ecu cx) were } \\
\text { undetected; Bloomia tropicalis (Blo } \\
\text { t), house dust mite allergens } 61 \\
\mathrm{CE} / \mathrm{g} \text { of dust (Der } \mathrm{p} 1 \text {, Der } \mathrm{f} 1 \text {, Der }\end{array}$ & - & - & $\begin{array}{l}\text { Daytime breathlessness, } \\
\text { respiratory infection, and } \\
\text { doctors diagnosed asthma }\end{array}$ \\
\hline
\end{tabular}




\begin{tabular}{|c|c|c|c|c|c|}
\hline & & & $\begin{array}{l}\text { m 1), and cockroach allergens was } \\
\text { low } 5 \mathrm{CE} / \mathrm{g} \text { of dust } \\
\text { Total fungal DNA } 1.76 \times 10^{5} \mathrm{CE} / \mathrm{g} \\
\text { of dust }\end{array}$ & & \\
\hline 14. & 19 & $\begin{array}{l}\text { School (7), Curitibia, } \\
\text { Brazil }\end{array}$ & 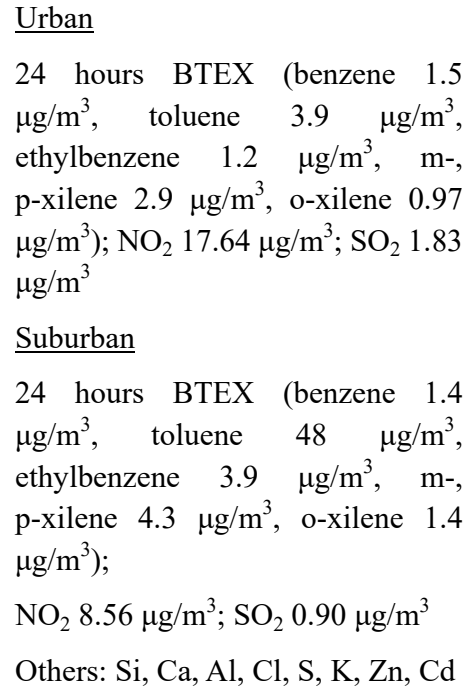 & 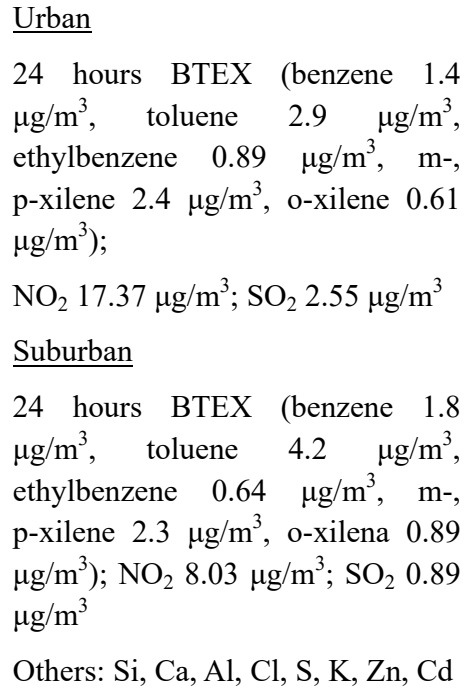 & $\begin{array}{l}\text { Location of school to the } \\
\text { source of pollutants } \\
\text { (traffic, petrochemical } \\
\text { industry), ventilation }\end{array}$ \\
\hline 15. & 20 & $\begin{array}{l}\text { School (1), Chennai, } \\
\text { India }\end{array}$ & $\begin{array}{l}\text { TSP }(24 \mathrm{~h}) 149.63 \pm 39.71-170.08 \\
\pm 55.28 \mu \mathrm{g} / \mathrm{m}^{3} ; \mathrm{Cl}^{-}(24 \mathrm{~h}) 3.18 \pm \\
1.98-3.65 \pm 2.65 \mu \mathrm{g} / \mathrm{m}^{3} ; \\
\text { Others: metal }(\mathrm{Al}, \mathrm{Fe}, \mathrm{Ca}, \mathrm{K}, \mathrm{Mg} \text {, } \\
\mathrm{Na}, \mathrm{Ba}, \mathrm{Cr}, \mathrm{Cu}, \mathrm{Mn}, \mathrm{Mo}, \mathrm{Ni}, \mathrm{Sr}, \mathrm{Ti} \text {, } \\
\mathrm{V}, \mathrm{Zn}) \text { and ion }\left(\mathrm{SO}_{4}{ }^{2+}, \mathrm{NH}_{4}^{+}, \mathrm{NO}_{3}{ }^{+} \text {, }\right. \\
\mathrm{Na}^{+}, \mathrm{Cl}^{-}, \mathrm{Ca}^{2+}, \mathrm{K}^{+}, \mathrm{Mg}^{2+}\end{array}$ & & $\begin{array}{l}\text { Location of school to the } \\
\text { source of pollutants - } \\
\text { (traffic) }\end{array}$ \\
\hline 16. & 21 & $\begin{array}{l}\text { School (1), Chennai, } \\
\text { India }\end{array}$ & $\begin{array}{l}\mathrm{PM}_{10}(8 \mathrm{~h}) 95 \pm 61-149 \pm 69 \\
\mu \mathrm{g} / \mathrm{m}^{3} ; \mathrm{PM}_{2.5}(8 \mathrm{~h}) 32 \pm 16-61 \pm 29 \\
\mu \mathrm{g} / \mathrm{m}^{3} ; \mathrm{PM}_{1}(8 \mathrm{~h}) 18 \pm 9-43 \pm 24 \\
\mu \mathrm{g} / \mathrm{m}^{3} ; \mathrm{CO}(8 \mathrm{~h}) 0.10 \pm 0.18-0.11 \pm \\
0.14 \mathrm{ppm} ; \mathrm{CO}_{2}(8 \mathrm{~h}) 307.38 \pm 21.25 \\
-387.77 \pm 44.97 ; \text { bacteria: } 443 \\
\mathrm{cfu} / \mathrm{m}^{3} ; \text { VOC was undetected }\end{array}$ & - & $\begin{array}{l}\text { Location of school to the } \\
\text { source of pollutants } \\
\text { (traffic), ventilation, and - } \\
\text { season }\end{array}$ \\
\hline
\end{tabular}




\begin{tabular}{|c|c|c|c|c|c|}
\hline 17. & 22 & $\begin{array}{l}\text { Primary school (1), } \\
\text { Alta Floresta, Mato } \\
\text { Grosso, Brazil }\end{array}$ & $\begin{array}{l}\text { Students } \\
(309, \quad 6-15- \\
\text { years })\end{array}$ & $\mathrm{PM}_{2.5}(24 \mathrm{~h}) 24.34 \pm 19.25 \mu \mathrm{g} / \mathrm{m}^{3}$ & 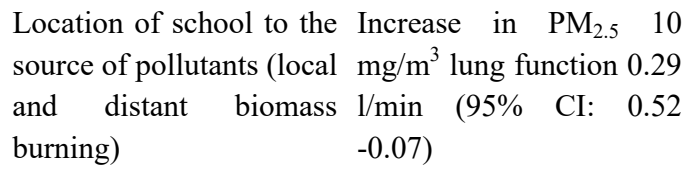 \\
\hline 18. & 23 & $\begin{array}{l}\text { High school (3), } \\
\text { Bhilai-Durg, } \\
\text { Chhattisgarh, India }\end{array}$ & $\begin{array}{l}\text { Students }(22, \\
16-18 \text { years }) ; \mathrm{PM}_{10}(24 \mathrm{~h}) 47.66 \pm 12.5-217.46 \pm \\
\text { Staff } \quad\left(30,17.2 \mu \mathrm{g} / \mathrm{m}^{3}\right. \\
25-55 \text { years })\end{array}$ & $\begin{array}{l}\mathrm{PM}_{10}(24 \mathrm{~h}) 36.4 \pm 8.75-97.4 \pm \\
12.2 \mu \mathrm{g} / \mathrm{m}^{3}\end{array}$ & $\begin{array}{l}\text { Location of school to the } \\
\text { source of pollutants - } \\
\text { (traffic and iron factory) }\end{array}$ \\
\hline
\end{tabular}

*numbers of school(s) studied 


\section{Discussion}

\subsection{Tropical School Air Quality}

Particulate matter (PM)

Particulate matter is one of the most common air pollutants which indicates the school's air quality. Studies in India reported a higher indoor PM concentration than outdoor; which exceeded both national air quality and WHO standards (Jan et al., 2017; Habil et al., 2015; Jyethi et al., 2014; Chithra \& Nagendra, 2013; Srimuruganandam \&Nagendra, 2011; Chithra \& Nagendra, 2012). Smaller size PM concentration was also found higher indoor (Chithra \& Nagendra, 2012). The indoor PM concentration found in Malaysian studies was also higher than outdoor and exceeding the WHO standards $\left(\mathrm{PM}_{10}\right)$, however, it was still under the national air quality threshold $\left(\mathrm{PM}_{10}, \mathrm{PM}_{2.5}\right)$. Meanwhile, studies in Brazil did not found a higher PM concentration $\left(\mathrm{PM}_{10}, \mathrm{PM}_{2.5}\right)$ which exceeded the air quality threshold. However, several schools reported a higher indoor PM concentration compared with outdoor.

Particulate matter composition and concentration was highly varied and depended on several factors, e.g., indoor and outdoor source of pollutants, outdoor weather, air exchange rate related with natural ventilation, penetration factor and deposition mechanism, and resuspension. Based on the articles that we studied, most schools were exposed to pollutant sources from outdoor (e.g., traffic road, industry) and indoor (e.g., building material, schoolroom furniture, and particulate matter attached to students' clothes). Rough PM ( $\left.\mathrm{PM}_{10}\right)$ concentration was significantly higher when the classroom was occupied. Smaller-sized PM $\left(\mathrm{PM}_{2.5}\right.$ dan $\left.\mathrm{PM}_{1}\right)$ were affected by the indoor and outdoor source of pollutants (Mohamad et al., 2016; Chithra \& Nagendra, 2014).

Several researches also did an analysis of chemical composition (i.e., metal and ion) of particulate matter that indicates the source of pollutants. In tropical countries with forests and plantations throughout the year, biomass burning activity was one of the most significant PM sources. Researches in Malaysia and Brazil found ion $\mathrm{K}^{+}$as one of PM components related to wood burning in residential area and farming activities (e.g., slash and burn land clearing method, burning of agriculture waste, especially from palm oil plantations (Mohamad et al., 2016; Habil et al., 2015).

The concentration of PM was also related to the seasonal variations. Researches in India have reported a higher indoor PM concentration during winter compared with summer. This was due to the low temperature and stable atmospheric condition during winter (Chithra \& Nagendra, 2012), changes in inhabitants' activity, and changes in the air exchange rate as a response to the weather change. In winter, resuspension of PM increased because students spend more time indoor and natural ventilation opening decreased, leading to PM indoor accumulation (Jan et al., 2017). Research on ambient air in India by Jyethi et al. (2014) reported a higher outdoor PM concentration in summer. The high concentration of $\mathrm{PM}_{10}$ can be explained by the high frequency of dust storm and crust resuspension in Delhi. Ambient $\mathrm{PM}_{10}$ concentration was predicted to be lower during the wet season, due to high humidity and high precipitation rate (Jyethi et al., 2014).

Total Suspended Particulate (TSP)

There were only two researches from Brazil and India which analyzed TSP pollutant. The increase in TSP concentration caused by pre-harvest sugarcane biomass burning was related to the increase of students' absence in Brazil during March until June. In India, TSP composition which indicates the source of pollutants was not related to indoor sources, but outdoor sources, i.e., fossil fuel burning activity, biomass burning, and sea salt (Chithra \& Nagendra, 2013).

The TSP concentration also showed seasonal variations with maximum concentration during winter due to the inversion condition and low mixing height appeared in that period which could cause a considerable amount of air pollutants to accumulate in the lower part of the atmosphere. Minimum TSP concentration was observed during summer due to better dispersion conditions, such as strong wind and temperature. In the wet season, the mean indoor daily TSP was lower. During this period, wind velocity became way lower compared to the other seasons. Low wind velocity resulted in a low rate of air exchange, which in turns supported the accumulation of indoor PM (Chithra \& Nagendra, 2013).

Nitrogen Dioxide $\left(\mathrm{NO}_{2}\right)$

Based on four articles studied, the concentration of indoor and outdoor $\mathrm{NO}_{2}$ in India, Malaysia, and Brazil was varied. Several locations showed a higher $\mathrm{NO}_{2}$ than WHO annual standards $40 \mu \mathrm{g} / \mathrm{m} 3$ (Jan R et al., 2017; Godoi et al., 2013; Norback et al., 2017; Raysoni et al., 2017). Research in Malaysia (Norback et al., 2017) showed that this high indoor $\mathrm{NO}_{2}$ concentration in classrooms was related to students' ocular symptoms and fatigue. The source of 
$\mathrm{NO}_{2}$ pollutant in this Malaysian study was fossil fuel burning and biomass burning from palm oil plantations (Norback et al., 2017).

Research in Brazil reported that the increase in $\mathrm{NO}_{2}$ concentration was related to the traffic emission and industrial activity (Godoi et al., 2013). Schools in the urban areas nearby a busy traffic area showed a higher concentration of $\mathrm{NO}_{2}$ compared with schools in the suburban areas. In India, indoor $\mathrm{NO}_{2}$ concentration exceeded the annual WHO standards and was higher in summer. There was no source of $\mathrm{NO}_{2}$ in the indoor school environment, therefore air current from the traffic activity outside was probably the main source of it. The concentration of $\mathrm{NO}_{2}$ coincides with the traffic activity, i.e., higher during morning and afternoon and almost constant during the day (Jan et al., 2017).

\section{Ozone $\left(\mathrm{O}_{3}\right)$}

We found only one article related to $\mathrm{O}_{3}$ in tropical schools during the past ten years. Research in India by Jan et al . (2017) reported that the indoor $\mathrm{O}_{3}$ concentration did not exceed WHO standards $100 \mu \mathrm{g} / \mathrm{m}^{3}$. Ozone gas was formed by sun-derived photochemical reactions and other gas pollutants (e.g., nitrogen oxide and VOC) in the atmosphere, therefore the presence of $\mathrm{O}_{3}$ inside a classroom was caused by airflow containing pollutant $\mathrm{O}_{3}$ from the outside (Jan et al., 2017; WHO, 2006). This explains why schools in the busy traffic area were exposed to indoor $\mathrm{O}_{3}$ which increased slowly after sunrise, reaching its maximum concentration during the day, and slowly decreasing in the afternoon. Besides that, the concentration of $\mathrm{O}_{3}$ was found positively correlated with the temperature. In summer months, the $\mathrm{O}_{3}$ concentration was higher because solar radiation is higher in summer than winter (Jan et al., 2017).

\section{Carbon Monoxide (CO)}

Researches on CO pollutant in tropical schools of Malaysia and India showed a small concentration of CO which did not exceed the national air quality standards. In every indoor school point of sampling, the CO concentration was always lower compared with the outdoor. The source of CO sampled was mainly incomplete combustion of fuel from the traffic vehicle and household waste burning in the surrounding school areas (Razali et al., 2015). The indoor CO concentration was only sampled during traffic peak hours (Chithra \& Nagendra, 2012). Results of researches in India showed a consistent trend with Malaysia, i.e., indoor CO concentration was always lower compared with outdoor. The CO concentration pattern was negatively correlated with the wind velocity, i.e., the higher wind velocity increased dispersion and diluted the ambient concentration which in turn lowering indoor $\mathrm{CO}$ concentration (Chithra \& Nagendra, 2012).

\section{Carbon Dioxide $\left(\mathrm{CO}_{2}\right)$}

Researches in Malaysia showed a higher indoor $\mathrm{CO}_{2}$ concentration, even though it did not exceed the national air quality standards. Researches in India showed contrary results; the indoor $\mathrm{CO}_{2}$ concentration was higher than 1000 ppm, exceeding the ASHRAE air quality standards (Jan et al., 2017). The observed concentration of $\mathrm{CO}_{2}$ showed a daily pattern with a higher concentration that coincides with the school active period (i.e., when students started to enter the classroom). This showed that the respiratory activity of the students being the main source of indoor $\mathrm{CO}_{2}$. Besides that, the airflow coming from outside the classroom also affected the indoor $\mathrm{CO}_{2}$ concentration. The fact that the indoor $\mathrm{CO}_{2}$ concentration did not exceed the air quality standard showed that the ventilation system has supported the air exchange, thus $\mathrm{CO}_{2}$ did not accumulate inside the classroom (Razali et al., 2015; Norback et al., 2017).

The study has also reported that the $\mathrm{CO}_{2}$ concentration was negatively correlated with the temperature and positively correlated with the air humidity. This found to be related to the openings in the ventilation system which allows air to be exchanged when the temperature changes, thus affect the relative indoor air humidity (Razali et al., 2015; Norback et al., 2017). Besides that, seasonal variations showed an increase of $\mathrm{CO}_{2}$ concentration in winter. During the winter, low wind velocity affects the airflow coming from the outside of the classroom, thus $\mathrm{CO}_{2}$ accumulates indoor. The main outdoor source of $\mathrm{CO}_{2}$ concentration found was biomass burning from the surrounding area (Razali et al., 2015).

Sulfur Dioxide $\left(\mathrm{SO}_{2}\right)$

Researches in India and Brazil have reported that the indoor and outdoor $\mathrm{SO}_{2}$ concentration was lower than WHO standards (i.e., $20 \mu \mathrm{g} / \mathrm{m}^{3}$ in 10 minutes or $500 \mu \mathrm{g} / \mathrm{m}^{3}$ in 24 hours). Considering that the main source of $\mathrm{SO}_{2}$ was fossil fuel burning and industrial activity, there was no significant correlation between the indoor and outdoor $\mathrm{SO}_{2}$ concentration at the urban school with high traffic activity and suburban school with petrochemical industrial activity (Godoi et al., 2013). The $\mathrm{SO}_{2}$ pollutant has a similar source and seasonal pattern as $\mathrm{CO}_{2}$ (Jan et al., 2017).

Volatile Organic Compounds (VOCs) 
Volatile Organic Compounds group consist of various hazardous carbon gas, however, the most frequently studied was BTEX (benzene; toluene; ethylbenzene; and o-, m-, p-xylenes). The BTEX is often found in gasoline and has a high solvability and toxicity, thus posing a high risk to human health. Research by Wesley et al., 2015. in Malaysia found no significant correlation between indoor and outdoor VOCs concentration at the schools in urban and suburban areas, however, the VOCs concentration was significantly correlated with the increase in students' respiratory inflammation (Wesley et al., 2015). Other research in Malaysia which specifically studied other indoor VOCs (i.e., formaldehyde, xylene, toluene, ethylbenzene, limonene, benzaldehyde, cyclohexane, methylcyclohexane, chlorobenzene, and para-dichloro-benzene) concluded that the source of those indoor VOCs was from the outdoor environment. Other research in Brazil with urban and suburban schools showed a consistent BTEX compound pattern. Both researches found a higher concentration of xylene and toluene compared with other detected VOCs (Norbäck et al., 2017; Godoi et al., 2013). The main source of VOCs found was incomplete fuel combustion, petrochemical industrial activity, and probably waste from farming and forest activity. Other rMalaysian studies have also reported the VOCs emission from palm oil plantation and forest (Tawfiq \&Aroua, 2016; Hewitt et al., 2009).

Polycarbon Aromatic Hydrocarbons (PAHs)

The source of PAHs pollutant found in the articles that we studied was incomplete fossil fuel and biomass burning. As shown by research in India, the source of PAHs in the school environment was incomplete fossil fuel and coal burning. The PAHs concentration was affected by changes in temperature, precipitation, and air humidity. The higher concentration of PAHs was shown in the winter period, followed by the wet season period, and the lowest in the summer (Jyethi et al., 2014).

Allergens and microorganisms

Exposure to airborne allergens and microorganisms will affect the immunity responses and caused respiratory symptoms. In the tropical countries with a constant warm temperature and high air humidity throughout the year, allergens (e.g., house dust mite) and microorganisms (e.g., fungi and bacteria) also present throughout the year. Research in Malaysia found a significant correlation between the presence of fungi Aspergillus and Streptomyces with the indoor air humidity (Norback et al., 2017; Wesley et al., 2015). Another research in Malaysia reported a low level of several allergens which dominated by house dust mite in the classroom dust (Norback et al., 2014).

\section{Health impact on students}

To understand health symptoms related to various pollutant exposure in the school environment, articles studied in this study have estimated the health risk, studied the health history, and measured biological indicators (e.g., FeNO and ECP).

Non-carcinogenic and carcinogenic health risk

Several researches showed that contaminated PM (by heavy metal and PAHs) can directly enter the human body through inhalation or direct contact with skin and oral, thus increasing the risk of diseases (i.e., cardiovascular, respiration, lung cancer), especially in school children which suffered from long-term exposure effect. Pollutant controlling program in the school is badly needed to counter this problem (Othman et al., 2019; Mohamad et al., 2016; Jyethi et al., 2014).

\section{Respiratory symptoms}

Respiratory symptoms were the most common health risk caused by air pollution. Researches studied reported the relationship between several respiratory problems with air pollutant agents. The exposure to PM related with various respiratory symptoms on students, especially in schools located nearby the source of pollutants (i.e., traffic, industry, biomass burning). Besides that, ocular symptoms, fatigue, headache, rhinitis, and SBS (Sick Building Syndrome) have been reported to be directly correlated with the indoor air quality which did not fulfill the requirements indicated by indoor exposure to $\mathrm{NO}_{2}$, VOCs and PM.

The prevalence of respiratory symptoms related to air pollutants was also measured by a certain biomarker (i.e., FeNO and ECP) to reduce the bias in health history which were gathered through students' questionnaire. Research in Malaysia with 386 students found the relation between the increase in FeNO ( $>20 \mathrm{pbb})$ concentration to respiratory symptoms which indicates lower airway inflammation due to mold Aspergillus exposure and the classroom dampness (Norback et al., 2017). Another research in preschools located in urban and suburban areas of Malaysia found that the increase in ECP which indicates upper airway inflammation was related to exposure to VOC, $\mathrm{PM}_{10}$, and indoor microorganisms (Wesley et al.,2015).

The health symptoms mentioned above have impacted the students' absence in school, especially younger students. 
Research in Brazil found that students' absence was related to the increase in PM concentration. These indicate that the poor air quality at school can affect the students' productivity and the quality of the education system in tropical countries.

\section{Conclusion}

The indoor school environment in the tropical countries has been exposed to various pollutants, i.e., particulate matter, nitrogen oxide, ozone, carbon dioxide, carbon monoxide, sulfur dioxide, volatile organic compounds, polycyclic aromatic carbons, allergens, and microorganisms. There were several environmental factors related to the pollutants' exposure, e.g., location of the school to the source of pollutants, ventilation system, building inhabitants, and meteorological factors related to the season. Several pollutants were exceeding the limit of air quality standards and directly related to several health symptoms, especially diseases related to the respiratory system. Even though some pollutants were under the air quality limit, several health symptoms still occurring. More importantly, exposure to pollutants has directly affected students' absences in school, thus control in pollutants at the school environment is urgently needed to protect the students' health and the education system's quality.

\section{Acknowledgments}

This study was supported by the funding from: Penelitian Dasar Unggulan Perguruan Tinggi Kementerian Riset, Teknologi, dan Pendidikan Tinggi Republic Indonesia Tahun Anggaran 2019 (Contract No. NKB-1 578/UN2.R3.1/HKP.05,00/2019).

\section{Competing Interests Statement}

There is no conflict of interest inflicted in this study.

\section{References}

Annesi-Maesano, I., Baiz, N., Banerjee, S., Rudnai, P., \& Rive, S. (2013). Indoor air quality and sources in schools and related health effects. J Toxicol Environ Heal - Part B Crit Rev., 16(8), 491-550, https://doi.org/10.1080/10937404.2013.853609

Bo, M., Salizzoni, P., Clerico, M., \& Buccolieri, R. (2017). Assessment of indoor-outdoor particulate matter air pollution: A review. Atmosphere (Basel), 8(12), 136, 1-18. https://doi.org/10.3390/atmos8080136

Chithra, V. S., \& Nagendra, S. M. S. (2013). Chemical and morphological characteristics of indoor and outdoor particulate matter in an urban environment. Atmos Environ., 77, 579-587. https://doi.org/10.1016/j.atmosenv.2013.05.044

Chithra, V. S., \& Nagendra, S. M. S. (2014). Impact of outdoor meteorology on indoor PM10, PM2.5 and PM1 concentrations in a naturally ventilated classroom. Urban Clim., 10, 77-91. https://doi.org/10.1016/j.uclim.2014.10.001

Chithra, V. S., Nagendra, S. M. S. (2012). Indoor air quality investigations in a naturally ventilated school building located close to an urban roadway in Chennai, India. Build Environ., 54, 159-167. https://doi.org/10.1016/j.buildenv.2012.01.016

Gadkari, N. M. (2010). Study of personal-indoor-ambient fine particulate matters among school communities in mixed urban-industrial environment in India. Environ Monit Assess., 165(1-4), 365-375. https://doi.org/10.1007/s10661-009-0952-3

Godoi, R. H. M., Godoi, A. F. L., Goncalves Junior, S. J., et al. (2013). Healthy environment - indoor air quality of Brazilian elementary schools nearby petrochemical industry. Sci Total Environ., 463-464, 639-646. https://doi.org/10.1016/j.scitotenv.2013.06.043

Habil, M., Massey, D. D., \& Taneja, A. (2015). Exposure from particle and ionic contamination to children in schools of India. Atmos Pollut Res., 6(4), 719-725. https://doi.org/10.5094/APR.2015.080

Hewitt, C. N., Mackenzie, A. R., Carlo, P. Di., Dorsey, J. R., \& Evans, M. (2009). Nitrogen management is essential to prevent tropical oil palm plantations from causing ground-level ozone pollution. Proceedings of the National Academy of Sciences, 106(44), 1-5. https://doi.org/10.1073/pnas.0907541106

Jacobson, L. D. S. V., Hacon, S. D. S., Castro, H. A. D., Ignotti, E., Artaxo, P., \& Leon, A. C. M. P. D. (2012). Association between fine particulate matter and the peak expiratory flow of schoolchildren in the brazilian subequatorial amazon: a panel study. Environmental Research, 117(none). https://doi.org/10.1016/j.envres.2012.05.006 
Jan, R., Roy, R., Yadav, S., \& Satsangi, P. G. (2017). Exposure assessment of children to particulate matter and gaseous species in school environments of Pune, India. Build Environ., 111, 207-217. https://doi.org/10.1016/j.buildenv.2016.11.008

Jyethi, D. S., Khillare, P. S., \& Sarkar, S. (2014). Risk assessment of inhalation exposure to polycyclic aromatic hydrocarbons in school children. Environ Sci Pollut Res Int., 21(1), 366-378. https://doi.org/10.1007/s11356-013-1912-6

Mauro, C. C., Ferrante, V. L. S. B., Arbex, M. A., Ribeiro, M. L., \& Magnani, R. (2015). Pre-harvest cane burning and health: the association between school absences and burning sugarcane fields. Rev Saude Publica, 49, 25. https://doi.org/10.1590/s0034-8910.2015049005641

Mohamad, N., Latif, M. T., \& Khan, M. F. (2016).Source apportionment and health risk assessment of PM10in a naturally ventilated school in a tropical environment. Ecotoxicol Environ Saf., 124, 351-362. https://doi.org/doi:10.1016/j.ecoenv.2015.11.002

Norbäck, D., Hashim, J. H., Hashim, Z., \& Ali, F. (2017). Volatile organic compounds (VOC), formaldehyde and nitrogen dioxide (NO2) in schools in Johor Bahru, Malaysia: Associations with rhinitis, ocular, throat and dermal symptoms, headache and fatigue. Sci Total Environ., 592, 153-160. https://doi.org/10.1016/j.scitotenv.2017.02.215

Norback, D., Hashim, J. H., Hashim, Z., \& Ali, F. (2017). Volatile organic compounds (VOC), formaldehyde and nitrogen dioxide (NO2) in schools in Johor Bahru, Malaysia: Associations with rhinitis, ocular, throat and dermal symptoms, headache and fatigue. Sci Total Environ., 592, 153-160. https://doi.org/10.1016/j.scitotenv.2017.02.215

Norback, D., Hashim, J. H., Hashim, Z., et al. (2017). Respiratory symptoms and fractional exhaled nitric oxide (FeNO) among students in Penang, Malaysia in relation to signs of dampness at school and fungal DNA in school dust. Sci Total Environ., 577, 148-154. https://doi.org/10.1016/j.scitotenv.2016.10.148

Norback, D., Markowicz, P., Cai, G.-H., et al. (2014). Endotoxin, ergosterol, fungal DNA and allergens in dust from schools in Johor Bahru, Malaysia- associations with asthma and respiratory infections in pupils. PLoS One, 9(2), e88303. https://doi.org/10.1371/journal.pone.0088303

Othman, Murnira, Latif, Mohd Talib, \& Matsumi, Yutaka. (2019). The exposure of children to pm2.5 and dust in indoor and outdoor school classrooms in kuala lumpur city centre. Ecotoxicology and Environmental Safety, 170(APR.), 739-749. https://doi.org/10.1016/j.ecoenv.2018.12.042

Raysoni, A. U., Armijos, R. X., Margaret Weigel, M., Montoya, T., Eschanique, P., \& Racines, M., et al. (2016). Assessment of indoor and outdoor pm species at schools and residences in a high-altitude ecuadorian urban center. Environmental Pollution, 214(jul.), 668-679. https://doi.org/10.1016/j.envpol.2016.04.085

Raysoni, A. U., Stock, T. H., Sarnat, J. A., Chavez, M. C., Sarnat, S. E., \& Montoya, T., et al. (2017). Evaluation of voc concentrations in indoor and outdoor microenvironments at near-road schools. Environmental Pollution, 231, 681-693. https://doi.org/10.1016/j.envpol.2017.08.065

Yang Razali, N. Y., Latif, M. T., Dominick, D., Mohamad, N., Sulaiman, F. R., \& Srithawirat, T. (2015). Concentration of particulate matter, co and co2 in selected schools in malaysia. Building and Environment, 87, 108-116. https://doi.org/10.1016/j.buildenv.2015.01.015

Rojas, J. C., Sánchez, N. E., Schneider, I., Oliveira, M. L. S., Teixeira, E. C., \& Silva, L. F. O. (2019). Exposure to nanometric pollutants in primary schools: Environmental implications. Urban Clim., 27, 412-419. https://doi.org/10.1016/j.uclim.2018.12.011

Sasso, F., Izard, M., Beneteau, T., et al. (2019). 18-year evolution of asthma and allergic diseases in French urban schoolchildren in relation to indoor air pollutant levels. Respir Med., 148, 31-36. https://doi.org/10.1016/j.rmed.2019.01.007

Srimuruganandam, B., \& Nagendra, S. M. S. (2011). Characteristics of particulate matter and heterogeneous traffic in the urban area of India. Atmos Environ., 45(18), 3091-3102. https://doi.org/10.1016/j.atmosenv.2011.03.014

Tawfiq, M. F., \& Aroua, M. K. (2016). Carbon Content Evaluation and Related Vocs Emission from Experimental Burn of Tropical Biomass Nik Meriam Nik Sulaiman. IOSR Journal of Applied Chemistry, 9(4), 51-56. https://doi.org/10.9790/5736-0904025156 
Wesley, A. D., \& Jalaludin, J. (2015). Indoor air pollutant exposure and eosinophil cationic protein as an upper airway inflammatory biomarker among preschool children. Procedia Environmental ences, 30, $297-302$. https://doi.org/10.1016/j.proenv.2015.10.053

WHO. (2006). WHO Air Quality Guidelines for Particulate Matter, Ozone, Nitrogen Dioxide and Sulfur Dioxide: Global Update 2005: Summary of Risk Assessment. https://doi.org/10.1016/0004-6981(88)90109-6

Yap, J., Ng, Y., Yeo, K. K., Sahlén, A., Lam, C. S. P., Lee, V., \& Ma, S. (2019). Particulate air pollution on cardiovascular mortality in the tropics: Impact on the elderly. Environ Heal A Glob Access Sci Source, 18(1), 1-9. https://doi.org/10.1186/s12940-019-0476-4

Zwozdziak, A., Sowka, I., Worobiec, A., Zwozdziak, J., \& Nych, A. (2015). The contribution of outdoor particulate matter (pm1, pm2.5, pm10) to school indoor environment. Indoor \& Built Environment, 24(8), 1038-1047. https://doi.org/10.1177/1420326X14534093

\section{Copyrights}

Copyright for this article is retained by the author(s), with first publication rights granted to the journal.

This is an open-access article distributed under the terms and conditions of the Creative Commons Attribution license (http://creativecommons.org/licenses/by/4.0/). 